\title{
Compliance with nedocromil sodium and a nedocromil sodium/salbutamol combination
}

\author{
G.L. Braunstein*, G. Trinquet**, A.E. Harper+, and a Compliance Working Group
}

Compliance with nedocromil sodium and a nedocromil sodium/salbutamol combination. G.L. Braunstein, G. Trinquet, A.E. Harper, and a Compliance Working Group. @ERS Journals Ltd 1996.

ABSTRACT: Nonadherence to prescribed preventive medication is common in asthma. We wanted to assess whether the combination of a $\beta_{2}$-bronchodilator with an anti-inflammatory treatment in the same metered-dose inhaler (MDI) with a regular dosing schedule might improve compliance.

A double-blind study was used to compare use (two actuations four times daily for 12 weeks) of $2 \mathrm{mg}$ nedocromil sodium ( $\mathrm{n}=101$ ) with a combination of $2 \mathrm{mg}$ nedocromil sodium and $100 \mathrm{\mu g}$ salbutamol $(n=100)$ in mild-to-moderate asthma patients (mean age $42 \pm 14$ yrs; 98 males and 103 females). Compliance was measured using the electronic Nebulizer Chronolog (NC) device, change in MDI canister weight, patient questionnaire and physician assessment.

The mean \pm SD number of actuations per day for nedocromil sodium and the combination during the primary period of assessment (Weeks 11-12) was 4.2 \pm 2.6 and 4.6 $\pm 2.5(\mathrm{NC}), 5.3 \pm 2.1$ and $5.3 \pm 2.0$ (canister weight), and 7.5 \pm 1.3 and $7.4 \pm 1.3$ (questionnaire), respectively. Physician assessment rated compliance as "good" to "excellent". The first and final days of the period were not used in the $\mathrm{NC}$ analysis to exclude part-days of treatment and drug-dumping (repeated actuations without inhalation), and may account for the difference between $\mathrm{NC}$ and canister weight results. The mean number of two-actuation doses per day determined from the NC was $2.1 \pm 1.3$ for nedocromil sodium and $2.4 \pm 2.1$ for the combination. Thirty five percent (nedocromil sodium) and $34 \%$ (combination) of the patients were compliant (6-10 actuations per day for $\geq 60 \%$ of the days).

We conclude that compliance is poor in asthma, electronic recording revealed the dumping phenomenon and, in this study, the combination of an inhaled $\beta_{2}$-bronchodilator with a preventive treatment did not improve compliance over a three month period in patients with mild-to-moderate asthma. Eur Respir J., 1996, 9, 893-898.
*Laboratoires Fisons S.A., Tour PFA, Paris la Défense, France. **6 rue Léonce Reynaud, Paris, France. +Fisons Pharmaceuticals, Respiratory Development, Loughborough, Leicestershire, UK.

Correspondence: G.L. Braunstein

Laboratoires Fisons S.A.

Tour PFA

La Défense 10

Cédex 43

F-92076 Paris la Défense

France

Keywords: Asthma

clinical trial

compliance

nedocromil sodium

salbutamol

Received: July 131995

Accepted after revision January 311996

This study was presented at the European Respiratory Society meeting, Barcelona, 16-20 September, 1995.
Nonadherence to prescribed medication is a major concern in the treatment of chronic disease $[1,2]$. It can result in medication being deemed ineffective or in administration of a larger dose than necessary for a therapeutic effect, thereby increasing the potential for adverse events. In asthma, compliance with preventive or maintenance therapy has been shown to be low [3]. Poor compliance may be associated with decreased asthma control $[4,5]$, and higher mortality [6].

Asthma treatment guidelines advocate the introduction of a maintenance inhaled anti-inflammatory drug (sodium cromoglycate, nedocromil sodium or low-dose inhaled corticosteroid) in moderate asthma when patients use inhaled $\beta_{2}$-bronchodilators more than three times per week to alleviate symptoms [7]. These latter drugs remain the most widely-used asthma treatment, however, and are still frequently prescribed for regular use. Compliance with regular anti-inflammatory treatment in these patients may be crucial, particularly if several daily doses are required to achieve efficacy.
One reason for poor compliance may be that patients do not perceive an immediate benefit from preventive treatments, whereas the immediate relief of symptoms after use of a bronchodilator may lead to patients preferring that treatment. We hypothesized that the combination of these two types of drug in the same metered-dose inhaler (MDI) canister might improve compliance with a regular regimen by associating an immediate benefit with inhalation of the preventive medication. A recently published study [8] addressed a similar question but the expected difference in compliance was based on simplifying the regimen: by replacing two canisters with one.

We have compared compliance with, and the patterns of use of nedocromil sodium and a combination of nedocromil sodium and salbutamol in mild-to-moderate asthma patients treated by general practitioners. Four methods of measuring compliance were used: electronic recording of actuations; change in canister weight; patient questionnaire; and physician assessment. 


\section{Methods}

\section{Study population}

Asthma patients aged 18-70 yrs were selected from primary care general practice. Mild-to-moderate asthma was defined as: acute and/or recurrent dyspnoea, cough or wheezing improved by an inhaled $\beta_{2}$-bronchodilator; a peak expiratory flow rate (PEFR) of $\geq 70 \%$ of predicted normal value for age, sex and height; and daily use of an inhaled $\beta_{2}$-bronchodilator. Patients were excluded if they had: used inhaled or systemic corticosteroids; experienced an acute exacerbation requiring hospitalization or emergency treatment within 2 months of the study; or had a medical history of more than one acute exacerbation requiring hospitalization, emergency treatment or systemic corticosteroid therapy per year.

\section{Study protocol}

Age, sex, and disease history data were collected on admission and patients were allocated, using a randomized code, to receive 12 weeks of treatment with either a nedocromil sodium MDI delivering $2 \mathrm{mg}$ per actuation (Tilade ${ }^{\circledR}$ ), or a combination MDI delivering $2 \mathrm{mg}$ nedocromil sodium and $100 \mu \mathrm{g}$ salbutamol per actuation (Zarent $(\mathrm{M})$ ), in addition to their current medication. Patients were instructed to inhale two actuations four times a day (morning, noon, afternoon and evening). Each canister contained 112 actuations; sufficient for 14 days of treatment. Patients were seen by the physician on entry and after 2, 10 and 12 weeks of treatment. Single canisters were dispensed at the first and third visits; and five canisters at the second visit. For Weeks 1-2 and Weeks 11-12, canisters were supplied fitted with a Nebulizer Chronolog (NC) device. This handheld electronic device (NC330; Medtrac Corp., Denver, CO, USA) contains a microprocessor and is compatible with an MDI mouthpiece and canister. It records the date and time of each actuation of the MDI (with a $3 \mathrm{~s}$ minimum recordable delay between successive actuations), and has the capacity to store data from over 1,000 actuations. Patients were informed that the $\mathrm{NC}$ registered drug delivery but were not informed that the date and time of each actuation were recorded. For the intervening 8 week period (Weeks 3-10), the canisters were supplied fitted with the Syncroner mouthpiece adapter (an open tube spacer) [9]. The patients returned their canisters at each clinic visit, when they received the appropriate instruction or reinforcement for inhaler and/or device use for the next period. They were not required to change or manipulate the canister and NC device or adapter.

The study was conducted double-blind, was in accordance to the Declaration of Helsinki, and was approved by an independent Ethics Committee. The protocol was as close as possible to normal practice to avoid any artificial improvement in compliance. All patients were informed of the study objective, of the nature of the treatment (anti-inflammatory or combined anti-inflammatory and bronchodilator) and gave their written informed consent before participating.
Measurement of compliance

Nebulizer Chronolog data. Analyses were based on a treatment day starting at $4.00 \mathrm{a} . \mathrm{m}$. and finishing at 4.00 a.m. the following day to account for late-night use. Data from the first and final day of each period were not used, in order to exclude part-days of treatment and drug-dumping. Daily compliance was the primary variable, and was defined as: perfect (eight actuations); good (6-10 actuations); underuse ( $<6$ actuations); or overuse ( $>10$ actuations). Secondary variables were: the number of actuations and doses per day; actuations per dose (number delivered within a 15 min interval); and the delay between doses. Actuations were also pooled by $1 \mathrm{~h}$ intervals and times of peak delivery were established. The pattern of delivery was then described from the number of actuations $\cdot 4 \mathrm{~h}^{-1}$ around the peak. Finally, patients were classified as compliant (at least $60 \%$ of days with 6-10 actuations $\cdot$ day $^{-1}$, i.e. at least $45 \%$ of total nominal dose) or noncompliant.

Weighing of canisters. All canisters were weighed before dispensing and on return. The number of actuations was calculated using a mean weight of $134 \mathrm{mg}$ per actuation for both treatments. The same electronic balance was used throughout (Mettler PM400; Mettler-Toledo, Greifensee, Switzerland).

Patient Questionnaire. At clinic visits during the treatment period, the patients were asked: how many actuations per day and per dose they inhaled; at what time during the day they took their treatment; and whether they tended to forget or take "more than", "less than" or "exactly" what had been prescribed.

Physician opinion. At each visit, the physician rated the compliance of the patient as: "excellent" (virtually no missed doses and a regular time interval between dosing); "good" (virtually no missed doses but irregular dosing); "moderate" (daily dosing but frequent, missed doses); and "poor" (seldom dosed).

\section{Statistical analysis}

The three treatment periods were analysed separately. The last period was the primary period. Compliance data were expressed as the mean \pm SD, and answers to the questionnaire and physician rating of compliance as percentage of patients. Treatment comparisons for the NC- and canister weight-derived variables, and patient questionnaire data were performed using Student's t-test. Comparisons between periods were performed using the paired t-test. Physician rating was analysed nonparametrically using the Mann-Whitney U-test. The relationship between change in weight and the NC data were studied by parametric regression. All tests were two tailed. A p-value of less than 0.05 was considered significant. 
Table 1. - Patient details

\begin{tabular}{lcc}
\hline & $\begin{array}{c}\text { Nedocromil } \\
\text { sodium }\end{array}$ & $\begin{array}{c}\text { Combination } \\
\text { therapy }\end{array}$ \\
\hline Patients n & 101 & 100 \\
Age yrs & $43 \pm 15$ & $41 \pm 14$ \\
Sex M/F & $48 / 53$ & $50 / 50$ \\
Duration of asthma yrs & $15 \pm 12$ & $15 \pm 12$ \\
Peak expiratory flow & & \\
$\quad$ L·min ${ }^{-1}$ & $443 \pm 72$ & $457 \pm 88$ \\
$\quad \%$ pred & $84 \pm 8$ & $85 \pm 10$ \\
$\begin{array}{l}\text { Diagnosis based on: } \\
\text { dyspnoea/cough/wheeze n }\end{array}$ & $70 / 57 / 74$ & $71 / 57 / 74$ \\
$\quad \beta_{2}$-induced reversibility of & 101 & 100 \\
$\quad$ symptoms n & & \\
Asthma treatment: & 101 & 100 \\
$\quad$ inhaled $\beta_{2} \mathrm{n}$ & $3 / 31$ & $4 / 23$ \\
$\quad$ oral $\beta_{2} /$ theophylline n & & \\
\hline
\end{tabular}

Values are presented as mean \pm SD. \% pred: percentage of predicted normal.

\section{Results}

Two hundred and one patients were included in the study: 101 received nedocromil sodium and 100 received the nedocromil sodium/salbutamol combination. There were no differences between the two groups for any pretreatment variable. Patients were mild-to-moderate asthmatics, bronchodilator users only, whose PEFR ranged $70-114 \%$ of predicted normal value (table 1). Eleven patients were withdrawn and no NC data were used. However, because the withdrawal of eight of these patients was related to treatment, they were included in the analysis with zero compliance (i.e. completely noncompliant). Seventy three NC could not be read because of battery failure, and for three patients treatment duration was less than 7 days and their data were disregarded (see table 2).

Treatment compliance from the NC data (the percentage of days against number of actuations per day) for the primary period is summarized in figure 1 . The percentage of days with perfect compliance ( 8 actuations $\cdot$ day $^{-1}$ ) was $14 \pm 20 \%$ with nedocromil sodium and $15 \pm 19 \%$ with the combination. For the first period of data collection (Weeks 1-2), the percentage of days with perfect compliance was $20 \pm 25 \%$ with nedocromil sodium and $18 \pm 23 \%$ with the combination. Zero, 4, 6 and 8 actuations.day ${ }^{-1}$ were the most common patterns of use, although this number ranged from 0 to $>14$ for both treatments (fig. 1). The remaining data on usage are presented in table 3 .

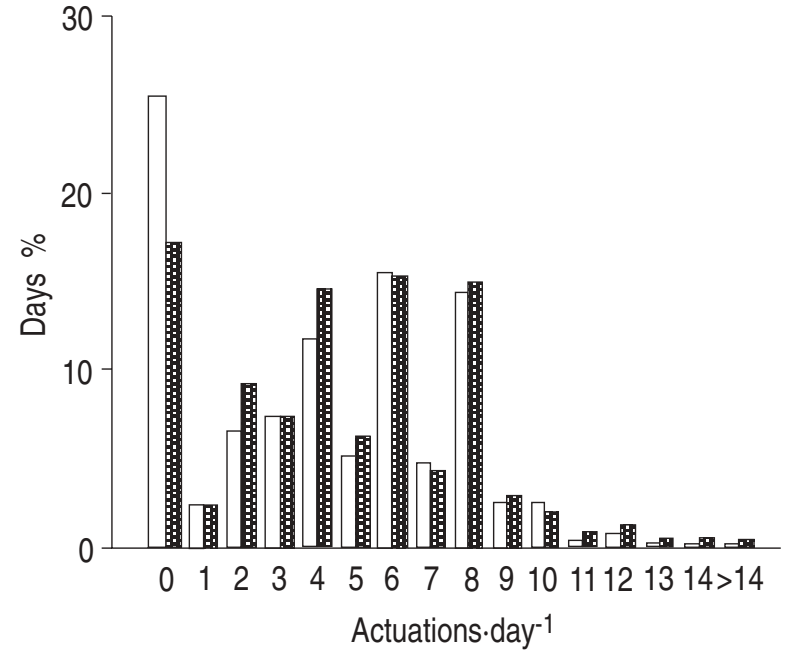

Fig. 1. - Nebulizer Chronolog treatment compliance data given as the mean percentage of days against the number of actuations.day-1 for the primary period, Weeks 11-12. $\square$ : nedocromil sodium; 眲明 : combination (nedocromil sodium/salbutamol)

There were no differences between treatments for any variable.

During Weeks 1-2 and 11-12, patients inhaled a mean \pm SD of 5.0 \pm 2.4 and $4.2 \pm 2.6$ actuations $\cdot$ day $^{-1}$ of nedocromil sodium and $4.7 \pm 2.3$ and $4.6 \pm 2.5$ actuations $\cdot$ day $^{-1}$ of the combined therapy, and administered a mean \pm SD of $2.6 \pm$ 1.2 and $2.1 \pm 1.3$ doses $\cdot$ day $^{-1}$ of nedocromil sodium and

Table 3. - Compliance assessed with the Nebulizer Chronolog

\begin{tabular}{|c|c|c|}
\hline & $\begin{array}{l}\text { Nedocromil } \\
\text { sodium }\end{array}$ & Combination \\
\hline \multicolumn{3}{|c|}{ Days with good use (6-10 actuations) \% } \\
\hline Weeks 1-2 & $48 \pm 36$ & $45 \pm 34$ \\
\hline Weeks 11-12 & $39 \pm 35$ & $40 \pm 33$ \\
\hline \multicolumn{3}{|c|}{ Days with perfect use (8 actuations)* \% } \\
\hline Weeks 1-2 & $20 \pm 25$ & $18 \pm 23$ \\
\hline Weeks 11-12 & $14 \pm 20$ & $15 \pm 19$ \\
\hline \multicolumn{3}{|c|}{ Days of underuse $(<6$ actuations) $\%$} \\
\hline Weeks 1-2 & $50 \pm 37$ & $53 \pm 35$ \\
\hline Weeks $11-12$ & $59 \pm 36$ & $57 \pm 35$ \\
\hline \multicolumn{3}{|c|}{ Days of overuse ( $>10$ actuations) $\%$} \\
\hline Weeks 1-2 & $3 \pm 8$ & $2 \pm 6$ \\
\hline Weeks $11-12$ & $2 \pm 6$ & $3 \pm 8$ \\
\hline
\end{tabular}

Values are presented as mean \pm SD. *: these data are a subset of "good use" (6-10 actuations).

Table 2. - Nebulizer Chronolog (NC) data available according to treatment period and treatment group (patient numbers)

\begin{tabular}{llcccc}
\hline Category & & \multicolumn{2}{c}{ Nedocromil sodium } & \multicolumn{2}{c}{ Combination } \\
A & Total $\mathrm{n}$ & Weeks $1-2^{101}$ & Weeks $11-12$ & Weeks 1-2 & Weeks 11-12 \\
& & 0 & 8 & 0 & 3 \\
\hline B & Withdrawn & 32 & 7 & 25 & 9 \\
C & NC battery failure & 0 & 6 & 0 & 2 \\
D & Compliance set to zero\# & 1 & 0 & 2 & 0 \\
E & data disregarded & 68 & 92 & 73 & 90 \\
& NC Data available* & 63 &
\end{tabular}

\#: compliance set to zero to account for treatment-related withdrawal (these patients are included in category B); : period of NC recording was less than 7 days; *: (A - B - C + D - E). 


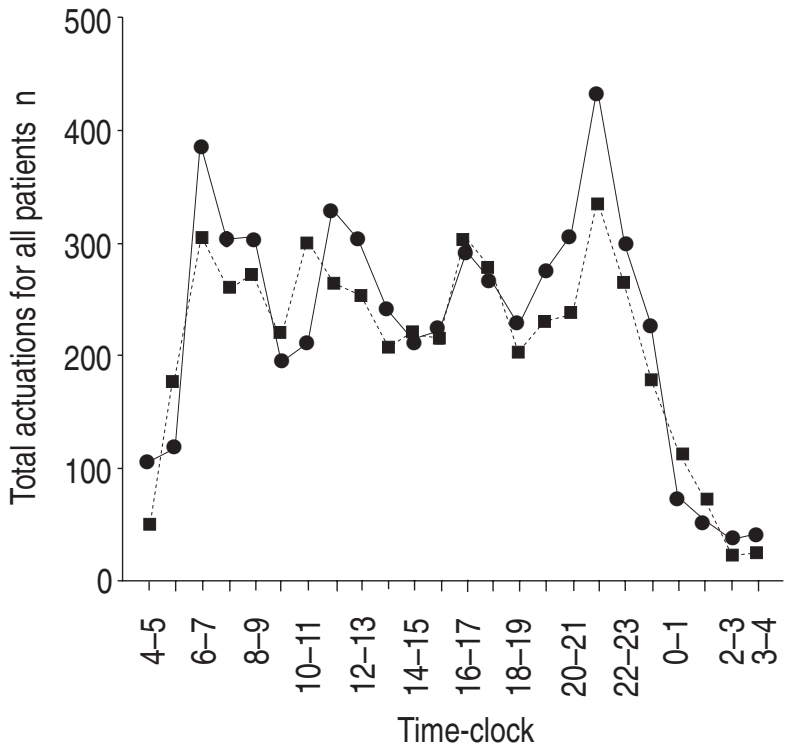

Fig. 2. - Nebulizer Chronolog treatment compliance data given as the number of actuation $\mathrm{h}^{-1}$ intervals for $24 \mathrm{~h}$ during the primary period, Weeks 11-12.

combination (nedocromil sodium/salbutamol).

$2.6 \pm 1.2$ and $2.4 \pm 1.2$ doses $\cdot$ day $^{-1}$ for the combined therapy. The number of actuations $\cdot$ day $^{-1}(\mathrm{p}<0.05)$ and number of doses day $^{-1}(\mathrm{p}<0.001)$ were significantly lower during weeks 11-12 compared with Weeks 1-2 with nedocromil sodium. Similar compliance was observed during both periods of treatment with the combined therapy. The mean delay between doses ranged from $359 \pm 118$ to $379 \pm 146$ min.

The distribution of actuations by $1 \mathrm{~h}$ intervals over $24 \mathrm{~h}$ during Weeks $11-12$ is shown in figure 2 . The pattern was similar for both treatments and for Weeks 1-2 and 11-12. Four peaks of drug delivery were identified,

\begin{tabular}{|c|c|c|c|c|c|c|c|c|c|}
\hline $\begin{array}{l}\text { a) Pati } \\
\text { Event }\end{array}$ & $\begin{array}{l}\text { ent Id: } \\
\text { To.: } 3\end{array}$ & & & & & Initiali & a d on & 121 & 92 at $12: 42$ \\
\hline $12 / 23 / 92$ & $18: 02$ & $21: 47$ & $21: 47$ & & & & & & \\
\hline $12 / 24 / 92$ & $08: 45$ & $08: 45$ & $12: 14$ & $12: 14$ & $16: 08$ & $16: 08$ & $20: 18$ & $20: 18$ & \\
\hline $12 / 25 / 92$ & 09:03 & 09:03 & $12: 21$ & $12: 21$ & $15: 56$ & $15: 56$ & $20: 15$ & $20: 15$ & \\
\hline $12 / 26 / 92$ & $09: 28$ & 09:28 & $12: 52$ & $12: 52$ & $16: 21$ & $16: 21$ & 20:08 & 20:08 & \\
\hline $12 / 27 / 92$ & $08: 22$ & $08: 22$ & $14: 37$ & $14: 37$ & $18: 46$ & $18: 46$ & $22: 25$ & $22: 25$ & $22: 25$ \\
\hline $12 / 28 / 92$ & 08:01 & 08:01 & $11: 36$ & $11: 36$ & $16: 13$ & $16: 13$ & $19: 57$ & $19: 57$ & \\
\hline $12 / 29 / 92$ & $07: 57$ & $07: 57$ & $14: 34$ & $14: 34$ & $21: 24$ & $21: 24$ & & & \\
\hline $12 / 30 / 92$ & 08:00 & 08:00 & $12: 25$ & $12: 25$ & $17: 22$ & $17: 22$ & $20: 47$ & $20: 47$ & \\
\hline $12 / 31 / 92$ & 08:01 & 08:01 & $12: 14$ & $12: 14$ & $16: 17$ & $16: 17$ & $19: 51$ & $19: 51$ & 19:51 \\
\hline $01 / 01 / 93$ & $08: 13$ & $08: 13$ & $15: 11$ & $15: 11$ & $18: 40$ & $18: 40$ & $22: 44$ & $22: 44$ & \\
\hline $01 / 02 / 93$ & 08:08 & 08:08 & $12: 19$ & $12: 19$ & $16: 13$ & $20: 27$ & $20: 27$ & $20: 27$ & \\
\hline $01 / 03 / 93$ & $07: 40$ & $07: 40$ & $12: 00$ & 12:00 & $15: 56$ & $19: 58$ & 19:58 & & \\
\hline $01 / 04 / 93$ & 08:04 & $08: 40$ & $12: 02$ & $12: 02$ & $16: 07$ & $16: 07$ & $19: 56$ & $19: 56$ & \\
\hline $01 / 05 / 93$ & $08: 15$ & $08: 15$ & $11: 52$ & $11: 52$ & $16: 05$ & $16: 05$ & $19: 56$ & $19: 56$ & \\
\hline $01 / 06 / 93$ & $07: 59$ & $07: 59$ & $11: 53$ & $11: 53$ & $15: 56$ & $15: 56$ & & & \\
\hline
\end{tabular}

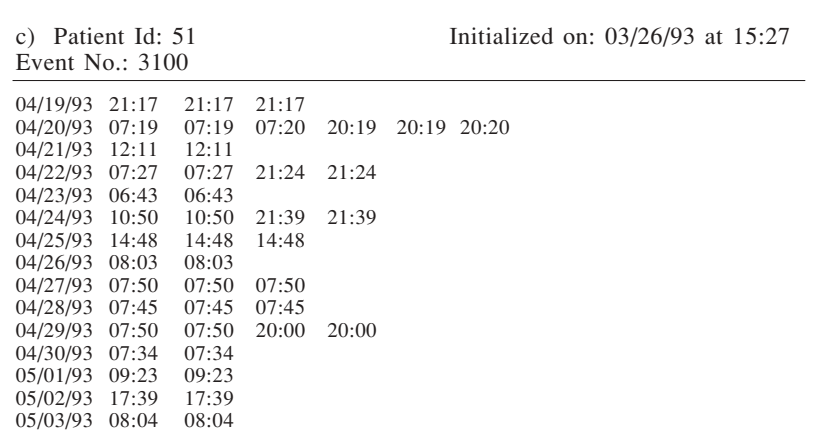

corresponding approximately to the required four times daily regimen: morning actuations were those delivered between 6.00 and 10.00 a.m.; noon actuations were delivered between 11.00 a.m. and 3.00 p.m.; afternoon between 4:00 and 8.00 p.m.; and evening between 9.00 p.m. and 1.00 a.m. The patients made a greater number of actuations during the morning and evening compared with noon and the afternoon. This difference was significant $(p<0.05)$ for the combination treatment during Weeks 11-12 (mean 1.9 actuations morning and evening, compared with 1.7 actuations noon and afternoon). The number of actuations morning and evening did not differ significantly between treatments.

Examination of individual $\mathrm{NC}$ data revealed several profiles of drug use. Very few patients regularly inhaled 8 actuations $\cdot$ day $^{-1}$ for the whole period (fig. 3a). Most of the patients either missed days (fig. 3b), or omitted actuations on several days (fig. 3c), or both, occasionally with a regular decrease over time (fig. 3d). Dumping was evident in some patients who fired a large number of actuations at the same time on the last day of the period (see also fig. 3b). During Weeks 1-2, $41 \%$ of patients in each treatment group were compliant. During Weeks $11-12,35 \%$ of patients were compliant with nedocromil sodium and $34 \%$ with the combination therapy.

The mean \pm SD number of actuations calculated from the change in canister weight was $5.4 \pm 2.1,6.3 \pm 2.7$ and $5.3 \pm 2.1$ actuations $\cdot$ day $^{-1}$ for Weeks 1-2, 3-10 and 11-12, respectively, for nedocromil sodium. For the combination therapy, the corresponding data were $5.4 \pm 2.0,6.3 \pm 2.5$ and $5.3 \pm 2.0$ actuations $\cdot$ day $^{-1}$. There was no significant difference between the treatments. The number of actuations calculated from the canister weight data was significantly higher than the number of actuations measured by the NC during Weeks $1-2$ on nedocromil sodium $(p<0.05)$ and on the combination therapy $(\mathrm{p}<0.01)$, and during Weeks $11-12(\mathrm{p}<0.001$ and $\mathrm{p}<0.01$, respectively). There was a

\begin{tabular}{|c|c|c|c|c|c|c|c|c|c|c|}
\hline $\begin{array}{l}\text { b) Patie } \\
\text { Event } N\end{array}$ & $\begin{array}{l}\text { ent Id: } \\
\text { [o.: } 40\end{array}$ & & & & & Initiali & ed on & $01 / 0$ & $193 \mathrm{a}$ & $15: 45$ \\
\hline $01 / 15 / 93$ & $19: 49$ & $19: 49$ & & & & & & & & \\
\hline $01 / 19 / 93$ & $17: 23$ & $17: 23$ & & & & & & & & \\
\hline $01 / 28 / 93$ & $18: 10$ & $18: 10$ & $18: 10$ & $18: 10$ & $18: 10$ & $18: 10$ & $18: 10$ & $18: 10$ & $18: 10$ & $18: 10$ \\
\hline & $18: 10$ & $18: 10$ & $18: 10$ & $18: 10$ & $18: 10$ & 18:10 & $18: 10$ & $18: 10$ & $18: 10$ & $18: 10$ \\
\hline & $18: 10$ & $18: 10$ & $18: 10$ & $18: 10$ & $18: 10$ & $18: 10$ & $18: 11$ & $18: 11$ & $18: 11$ & $18: 11$ \\
\hline & $18: 11$ & $18: 11$ & $18: 11$ & $18: 11$ & $18: 11$ & 18:11 & $18: 11$ & $18: 11$ & $18: 11$ & $18: 11$ \\
\hline & $18: 11$ & $18: 12$ & $18: 12$ & $18: 12$ & $18: 12$ & & & & & \\
\hline
\end{tabular}

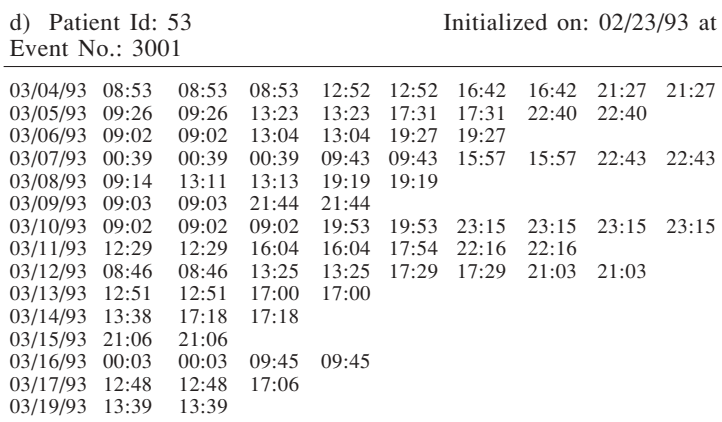

Fig. 3. - Examples of Nebulizer Chronolog (NC) data for individual patients. a) 8 actuations $\cdot$ day ${ }^{-1}$ on most days; b) missed days (including the "dumping" phenomenon); c) omitted actuations; d) regular decrease with use over time. 
Table 4. - Compliance assessed by patient questionnaire

\begin{tabular}{lcc}
\hline & $\begin{array}{c}\text { Nedocromil } \\
\text { Sodium }\end{array}$ & Combination \\
\hline${\text { Actuations } \cdot \text { day }^{-1}}^{-1}$ & $7.5 \pm 1.3$ & $7.4 \pm 1.3$ \\
Actuations $^{-10 \text { dose }^{-1}}$ & $2.0 \pm 0.3$ & $2.0 \pm 0.3$ \\
Dosing habits: \% patients taking & & \\
morning/evening doses & $99 / 98$ & $99 / 98$ \\
noon/afternoon doses & $88 / 87$ & $85 / 85$ \\
occasionally forgetting doses & 60 & 60 \\
more than/less than/as prescribed & $1 / 42 / 57$ & $6 / 34 / 60$ \\
\hline
\end{tabular}

Values are presented as mean \pm SD.

highly significant correlation between change in canister weight and NC data both during Weeks 1-2 (nedocromil sodium: $\mathrm{r}=0.81$; and combination: $\mathrm{r}=0.69 ; \mathrm{p}<0.001$ ) and Weeks 11-12 (nedocromil sodium: $r=0.70$; and combination: $\mathrm{r}=0.70 ; \mathrm{p}<0.001)$.

Results from the patient questionnaire during the final visit are given in table 4. Results were similar between visits. Although patients admitted that they missed actuations, most claimed they were taking their treatment morning, noon, afternoon and evening. Approximately two thirds of the patients believed they were taking exactly what was prescribed, whereas approximately one third declared they were taking less than prescribed. Data concerning the number of daily actuations were significantly higher than canister weight and NC data during the first and final 2 week periods for both treatments $(p<0.001)$. There was no significant difference in the number of actuations per dose as assessed by questionnaire and by the NC.

In the opinion of the physicians, $75 \%$ (compared with $76 \%$ at the end of week 2) of the nedocromil sodiumtreated patients and $76 \%$ (compared with $85 \%$ at the end of Week 2) of the combination-treated patients were good or excellent compliers with the dosing schedule at the end of Week 12. The remainder were moderate or poor compliers. Fewer patients were considered good or excellent compliers during Weeks 3-10 (58\% for the nedocromil sodium and $72 \%$ for the combination-treated patients) compared with Weeks 1-2 or 11-12. There were no significant differences between treatments.

\section{Discussion}

Poor compliance to treatment is common in patients with asthma. Our hypothesis was that the patients' perception of an immediate improvement in symptoms as a result of inhaling a bronchodilator would increase their overall compliance with, and compared to, the preventive drug, nedocromil sodium. This did not seem to be the case. Our results, therefore accord with those studies that have shown overall poor compliance with preventive and bronchodilator drugs administered as single treatments [10-12], and with compliance to a combination of an inhaled corticosteroid and $\beta_{2}$-bronchodilator administered via the Turbuhaler® device [8]. Neither the use of a single device, instead of two inhalers containing the individual drugs [8], nor the use of one device irrespective of treatment (our study) improved comparative compliance.
Compliance with asthma therapies seems to be independent of the severity of the disease [13], and the level of asthma control can differ between patients on identical regimens as a result of differing compliance [5]. Compliance has, therefore, been described as patient-rather than drug-dependent [11], and as "not a symptom-driven behaviour" [8]. This aspect has been described both for bronchodilator [14] and preventive medications [11, 13], irrespective of the method of measurement, and may explain our findings and those of BosLey et al. [8]. It may also be that the patients did not have sufficiently severe asthma to perceive a benefit from the bronchodilator, or that use of rescue inhaled bronchodilator masked this potential benefit. This lack of a relationship between perception of asthma and level of obstruction has also been described [15].

In our study, four measures of compliance were used. Canister weight and questionnaire data overestimated compliance compared with the electronic Nebulizer Chronolog recordings, with the latter identifying the lowest level of compliance with treatments. It seems probable that the near perfect compliance determined from the questionnaire was a result of the patients' desire to appear to adhere to their doctors' recommendations. Evaluation by physicians was the least accurate of the four methods, and lends confirmation to the idea that doctors tend to overestimate compliance [2].

We are not aware of other studies that have fully employed these methods of assessment of drug compliance, although work has been published on patterns of inhaler use and of the dumping [12] or MSA (multiple simultaneous activations) [16] phenomenon. By excluding the final day of Chronolog data, we were able to exclude the days with dumping. This was not possible for the canister weight assessment of compliance and may explain the discrepancy we found between the two methods of measurement. In the absence of pharmacokinetic analyses, recorded canister actuations cannot be considered as known inhalations; hence, although we tried to design a study as close as possible to normal general practice, the level of compliance described by the Chronolog data is likely to be an optimistic view of true compliance in general practice. This method of recording does, however, define the profile of use in terms of timing of actuations, and despite suboptimal compliance with both treatments, for patients on the combination therapy, this was mainly a result of missed actuations during the daytime, with the number of morning and evening actuations being close to ideal. Patients using the combination therapy had less days with no actuations delivered and, from the comparison within treatments, had more stable compliance with time than users of nedocromil sodium alone.

With $34-41 \%$ of patients taking $\geq 45 \%$ of the prescribed medication, the level of compliance was similar to or lower than that reported in previous studies: only $33 \%$ of patients in general practice [17], and 57\% of patients monitored in an asthma clinic [18], were compliant with their medication (defined as $\geq 50 \%$ use of the prescribed amount). Compliance has been shown to be higher in patients treated by specialists than by general practitioners, possibly as a result of better patient education [17]. If education is focused, (i.e. explanation of how a treatment works and why it is necessary to adhere to a particular regimen [18]), however, it should be possible 
to improve compliance in all treatment settings. This suggests that, although the patients in our study had agreed to take part and were co-operative to the overall aims of the protocol, the absence of positive intervention was more influential on compliance than the type of drug. The hypothesized preference for a $\beta_{2}$-bronchodilator was not reflected in improved compliance and tends to corroborate the supposition that the patient is the main variable.

A major consequence of poor compliance is an increase in morbidity. This may be difficult to evaluate in mild-to-moderate asthma, mainly because patients with long-standing disease may not be aware of a potential improvement with new therapy. We should, however, expect poor compliance of our patients and forestall its occurrence. As our study was carried out by general practitioners within the primary care setting, it is likely that our results are relevant to the vast majority of asthma patients.

\section{Compliance Working Group}

D. Amsellem (Valenton), J. Baracchini (Massy), Ch. Baranes (Paris), Ph. Barlet (Paris), H. Belahcen (Paris), A. Benhamou (Paris), G. Boublil (Saint-Ouen), B. Brami (Montreuil), Cl. Brugeas (Massy), F. Cajfinger (Verneuil s/S), R. Casassus (Ablon), M. Chelly (Ablon), D. Chevallier (Arnouville les Gonesses), B. Cohen (Massy), M. Cohen (Paris), R. Dahan (Aulnay s/Bois), M. Delanerie (Choisy le Roi), R. Demma (Drancy), G. Dennewald (Saint Denis), N. Elfassy (Paris), J. Fridman (Paris), P. Gamain (Valenton), D. Haas-Duchez (Paris), G. Hayoun (Drancy), S. Houdry (Paris), J.C. Ingrand (Paris), P. Jacob (Paris), F. Job (Massy), C. Jourdheuille-Nguyen Nhu (Valenton), E. Kaufman (Paris), B. Labatut (Paris), J.P. Lacoste (Paris), M. Laurent (BlancMesnil), E. Leriche-Notarianni (Levallois), J.A. Letourmy (Cachan), J.E. Levy (Saint-Ouen), E. Malarmey (Neuilly s/S.), A. Meyer (Massy), S. Mimran (Saint Ouen), H. Nguyen Nhu (Vigneux), M.F. Rancourt (Drancy), J. Rebot (Epinay s/Orge), P. Richard (Valenton), R. Rolland (Livry-Gargan), G. Trinquet (Paris), Ph. Turbie (Paris), L. Van Nguyen Anh (Massy).

\section{References}

1. Sackett DL, Snow JC. The magnitude of compliance and noncompliance. In: Haynes RB, Taylor DW, Sackett DL, eds. Compliance in Health Care. Baltimore, Johns Hopkins University Press, 1979; pp. 9-25.

2. Wright EC. Noncompliance - or how many aunts has Matilda? Lancet 1993; 342: 909-913.

3. Horn CR. Compliance by asthma patients: How much of a problem? Res Clin Forum 1986; 8: 47-53.

4. Cluss PA, Epstein LH, Galvis SH, Fierman P, Friday G.
Effects of compliance for chronic asthmatic children. $J$ Cons Clin Psychol 1984; 52: 909-910.

5. Horn CR, Clark TJH, Cochrane GM. Compliance with inhaled therapy and morbidity from asthma. Respir Med 1990; 84: 67-70.

6. Birkhead G, Attaway NJ, Strunk RC, Townsend MC, Teusch S. Investigation of a cluster of deaths of adolescents from asthma: evidence implicating inadequate treatment and poor patient adherence with medications. J Allergy Clin Immunol 1989; 84: 484-491.

7. National Heart, Lung, and Blood Institute, National Institutes of Health, Bethesda, Maryland. International consensus report on the diagnosis and treatment of asthma. Eur Respir J 1992; 5: 601-641.

8. Bosley CM, Parry DT, Cochrane GM. Patient compliance with inhaled medication: does combining beta-agonists with steroids improve compliance? Eur Respir $J$ 1994; 7: 504-509.

9. Newman SP, Clark AR, Talaee N, Clarke SW. Pressurised aerosol deposition in the human lung with and without an "open" spacer device. Thorax 1989; 44: 706-710.

10. Zora JA, Lutz CN, Tinkelman DG. Assessment of compliance in children using inhaled beta-adrenergic agonists. Ann Allergy 1989; 62: 406-409.

11. Dompeling E, Van Grunsven PM, Van Schayck CP, Folgering H, Molema J, Van Weel C. Treatment with inhaled steroids in asthma and chronic bronchitis: long term compliance and inhaler technique. Fam Pract 1992; 9: 161-166.

12. Rand CS, Wise RA, Nides M, et al. Metered-dose inhaler adherence in a clinical trial. Am Rev Respir Dis 1992; 146: 1559-1564.

13. Mann MC, Eliasson O, Patel K, ZuWallack RL. An evaluation of severity-modulated compliance with q.i.d. dosing of inhaled beclomethasone. Chest 1992; 102: 1342-1346.

14. Eney RD, Goldstein EO. Compliance of chronic asthmatics with oral administration of theophylline as measured by serum and salivary levels. Pediatrics 1976; 54: 513-517.

15. Orehek J, Beaupré A, Badier M, Nicloi MM, Delpierre S. Perception of airway tone by asthmatic patients. Bull Europ Physiopath of Respir 1982; 18: 601-607.

16. Mawhinney H, Spector SL, Kinsman RA, et al. Compliance in clinical trials of two nonbronchodilator, antiasthma medications. Ann Allergy 1991; 66: 294-299.

17. Dekker FW, Dieleman FE, Kaptein AA, Mulder JD. Compliance with pulmonary medication in general practice. Eur Respir J 1993; 6: 886-890.

18. Smith NA, Seale P, Ley P, Shaw J, Bracs PU. Effects of intervention on medical compliance in children with asthma. Med J Aust 1986; 144: 119-122. 